\title{
Distributed Address Assignment with Address Borrowing for ZigBee Networks
}

\author{
Shu-Chiung Hu*, Cheng-Kuan Lin ${ }^{\dagger}$, Yu-Chee Tseng ${ }^{* \ddagger}$, Wen-Tsuen Chen ${ }^{\ddagger}$ \\ *Department of Computer Science, National Chiao Tung University, Hsinchu, 30010, Taiwan \\ ${ }^{\dagger}$ School of Computer Science and Technology, Soochow University, Suzhou, 215006, China \\ $\ddagger$ Institute of Information Science, Academia Sinica, Taipei, 11529, Taiwan
}

\begin{abstract}
ZigBee is a popular communication standard for wireless sensor networks. It suggests a distributed address assignment mechanism (DAAM), which is easy to implement but may restrict the number of children of a router and the depth of the network. Since DAAM is too conservative, the utilization of the 16-bit address pool may become poor, causing the orphan problem. In this paper, we propose a distributed address assignment scheme by allowing a parent to borrow a subtree of address space from a neighbor to alleviate the orphan problem. When a new node tries to associate with a parent router which has no free address, this parent router will inquire its 2 hop neighbors for lending a subtree of free address space. We also propose a light-weight routing to support one-to-one routing in this environment.
\end{abstract}

Index Terms - addressing, address borrowing, routing, wireless sensor network, ZigBee

\section{INTRODUCTION}

Wireless sensor networks (WSNs) have attracted a lot of attention. Recently, research efforts have been dedicated to power management [9], routing [6], deployment and coverage [2], and localization [1]. Nowadays, many WSN systems have adopted ZigBee [10] as their communication protocol. ZigBee adopts the physical (PHY) and the medium access control (MAC) layers defined by IEEE 802.15.4 [5] and extends to network, application, and security services. ZigBee supports three network topologies, star, tree, and mesh. Regardless of network topology, there is a coordinator responsible for initializing, maintaining, and controlling the network. Star networks can only cover small areas, but tree and mesh networks can cover larger fields by allowing multi-hop communications. The backbone of a tree/mesh network is formed by one coordinator and multiple routers. An end device must associate with the coordinator or a router. In a tree network, routing can be done in a stateless manner based on nodes' 16 -bit short addresses.

To form a ZigBee network, addressing is the first work to be done. ZigBee suggests a distributed address assignment mechanism (DAAM), which enforces some addressing rules. By this mechanism, the coordinator needs to decide three parameters: the maximum number of children of a router $\left(C_{\max }\right)$, the maximum number of child routers of a router $\left(R_{\max }\right)$, and the maximum network depth $\left(L_{\max }\right)$. While simple, the scheme may prohibit a node from accepting isolated child routers/devices. A node is an orphan node when it cannot associate with any parent router but there is still unused address space in the network. Some heuristics algorithms are proposed to reduce orphans [7].

Reference [3] proposes an address borrowing scheme. When a new node sends an association request to a router of a ZigBee network and this router does not have any free address, it will ask other neighboring routers to lend an unassigned address to serve the new node. Park et al. [8] propose a distributed borrowing addressing scheme $(D B A S)$ by allowing a parent node to borrow a maximum unused address space to alleviate the orphan problem.

The main goal of our work is to alleviate the orphan problem by a distributed address assignment scheme with address borrowing. We improve the borrowing scheme of [8] by allowing a parent router to borrow a flexible subtree of address space from a neighbor. We also define the detailed procedure for our address-borrowing scheme, which is compatible with the ZigBee standard. Our solution solves addressing and routing issues altogether. When a new node tries to associate with a parent router with no unassigned address, the new node will estimate the number of orphan nodes in its neighborhood and this parent router will ask its 2-hop neighbors to lend a suitable address space to serve the new node. Then this new node can use the ZigBee DAAM to serve its children. We also show that routing and maintaining the borrow and lend lists can be easily done.

\section{OVERVIEW OF IEEE 802.15.4 AND ZigBeE STANDARDS}

IEEE 802.15.4 [4] specifies the physical and data link protocols for low-rate wireless personal area networks ( $L R$ $W P A N)$. IEEE 802.15.4 devices are classified as full function devices (FFDs) and reduced function devices (RFDs). IEEE 802.15.4 supports star and peer-to-peer topologies. In each personal area network $(P A N)$, one device is designated as the coordinator, which is responsible for maintaining the network. A FFD has the capability of serving as a coordinator or associating with an existing coordinator/router and becoming a router. A RFD can only associate with a coordinator/router and cannot have children.

In ZigBee, address assignment is done in a distributed manner. To form a network, the coordinator determines $C_{\max }$, $R_{\max }$, and $L_{\max }$ first. Note that the children of a router include both routers and end devices. So $C_{\max } \geq R_{\max }$ and up to $C_{\max }-R_{\max }$ children must be end devices. Addresses are assigned in a top-down manner. The coordinator takes 0 


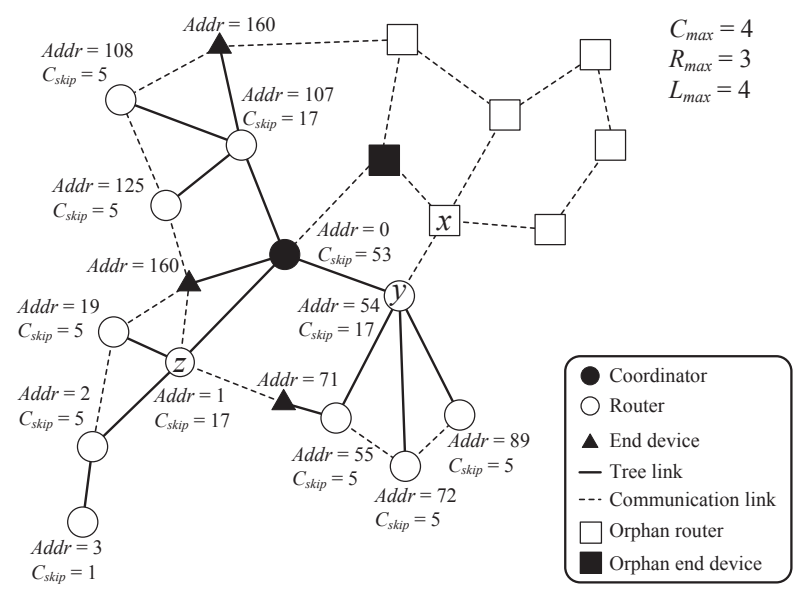

(a)

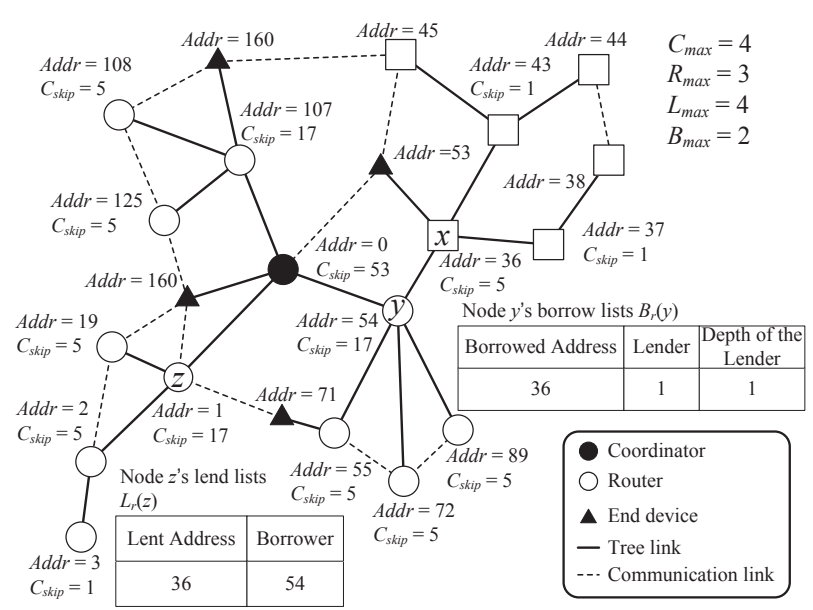

(b)

Fig. 1. An example of the ZigBee tree formed by (a) the original ZigBee DAAM and (b) our address-borrowing scheme.

as its address and divides the remaining address space into $R_{\max }+1$ blocks. The first $R_{\max }$ blocks are to be assigned to its child routers and the last block has $C_{\max }-R_{\max }$ addresses, each to be assigned to one child end device. The similar process is adopted by each child router to partition its given address space in a recursive manner. From $C_{\max }, R_{\max }$, and $L_{\max }$, each router at depth $d$ can compute a $C_{\text {skip }}(d)$ value, which is the size of one address block to be assigned to a child router [10]:

$C_{s k i p}(d)=\left\{\begin{array}{l}1+C_{\max } \cdot\left(L_{\max }-d-1\right) \quad \text { if } R_{\max }=1 \\ \frac{1+C_{\max }-R_{\max }-C_{\max } \cdot R_{\max } L_{\max }-d-1}{1-R_{\max }} \text { otherwise. }\end{array}\right.$

The value of $d$ is 0 for the coordinator and is increased by one as we go down the tree.

\section{The Proposed Addressing Scheme}

\section{A. Basic Idea}

Fig. 1(a) shows a ZigBee address assignment example. Router $y$ cannot accept router $x$ as its child because it already has the maximum $R_{\max }=3$ child routers, making $x$ an orphan node. The orphan problem [7] refers to the scenario where some nodes in Fig. 1(a) cannot connect to the network even though there are free addresses in the network.

We try to alleviate the orphan problem by allowing addressborrowing. We define a new Borrowing Information Base $(B I B)$ attribute called MaxBorrowingNumber $\left(B_{\max }\right)$, which can be carried by any reserved field. $B_{\max }$ is the maximum number of addresses that a router can borrow. A router can serve up to $R_{\max }+B_{\max }$ child routers and up to $C_{\max }-R_{\max }+B_{\max }$ child end devices. Here, these $B_{\max }$ children can be routers or end devices. Fig. 1(b) shows an example where $y$ has borrowed the address block rooted at 36 of depth 2 from router $z$. It also shows that the address block is assigned to router $x$. Then router $x$ can assign them to its children.
Below, we propose a fully automated, distributed ZigBee address assignment scheme to facilitate such borrowing behaviors. Then we further show how to conduct address-based routing in the network.

\section{B. Address-Borrowing Scheme (ABS)}

1) Variables: During the formation process, each node $x$ maintains the following variables:

- $A_{x}$ : the address of $x$ in a ZigBee network.

- $R(x)$ and $E(x)$ : the child routers and the child end devices currently associating with $x$, respectively.

- $L_{r}(x)$ and $L_{e}(x)$ : the lend lists of child routers' addresses and child end devices' addresses, respectively, which $x$ has lent out.

- $B_{r}(x)$ and $B_{e}(x)$ : the borrow lists of child router's addresses and child end device's addresses, respectively, which has borrowed.

- $p(x)$ : the address of $x$ 's parent in a ZigBee network.

- $o(x)$ : the estimated number of orphans in $x$ 's neighborhood.

- $d_{x}$ : the depth of $x$ in the ZigBee network. Note that there are two interpretations for the value of $d_{x}$. Under the normal situation, $d_{x}$ is the actual depth value of $x$. However, $d_{x}$ is the "depth" of the address counting from its original location (not $x$ 's location). For example, the $d_{z}$ of $z$ in Fig. 2 is 1 since originally address 107 is the child of address 0 (the coordinator $t$ ) even though its current physical depth in the ZigBee tree is 4. Similarly, the $d_{y}$ of $y$ in Fig. 2 is 2 since it is the child of $z$, and its physical depth in the ZigBee tree is 5 .

- $s_{x}$ : the state of $A_{x}$ 's location. We allow an address block to lent out if it is not a borrowed address black. Note that a child address block of a "borrowed" address block is also considered a "borrowed" address block. That is, a borrowed address block connot be further lent out to other nodes, and so is its sub-blocks. We set $s_{x}=$ "original" if $A_{x}$ is not a borrowed address; otherwise, we set $s_{x}=$ 
"borrowed". For example, in Fig. 2, $s_{z}=$ "borrowed" since $A_{z}=107$ is borrowed from the coordinator. Since $A_{y}$ is a child of $A_{z}, s_{y}=$ "borrowed", too. On the other hand, $A_{w}$ is not a borrowed address, so $s_{w}=$ "original".

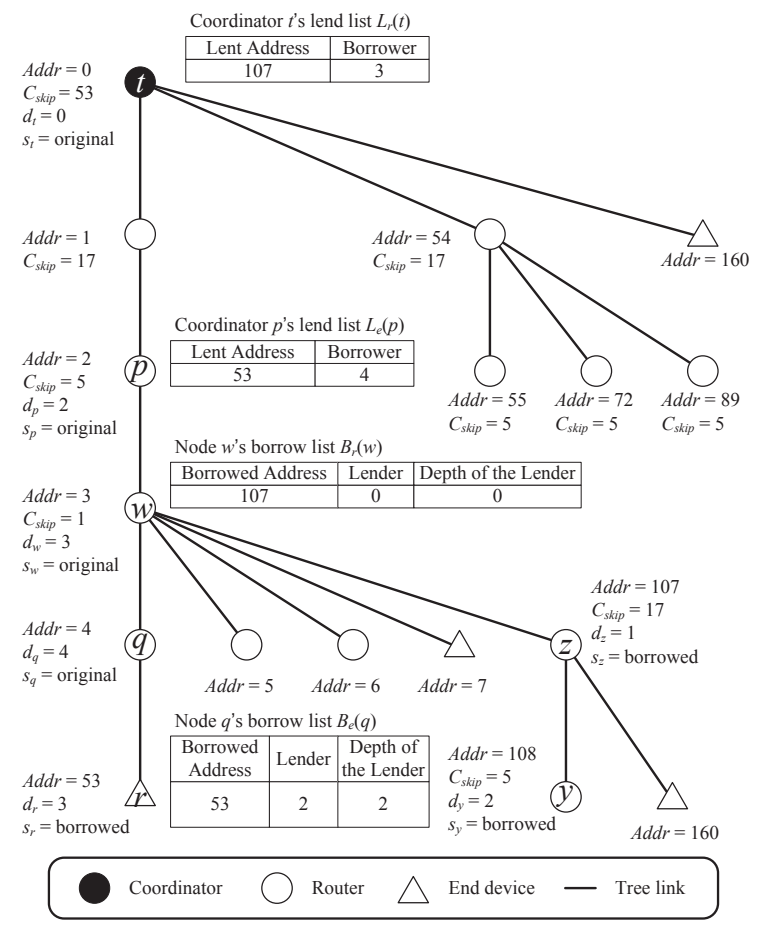

Fig. 2. An example of depth in the ZigBee tree $Z$ formed by ABS with $C_{\max }=4, R_{\max }=3, L_{\max }=4$, and $B_{\max }=2$.

2) Associating Scheme: To start a new network, a coordinator $t$ first sets the following parameters: $C_{\max }, R_{\max }, L_{\max }$, and $B_{\max }$. Then $t$ assigns $A_{t}=0, d_{t}=0$, and $s_{t}=$ "original", and sets $R(t), E(t), B_{r}(t), B_{e}(t), L_{r}(t)$, and $L_{e}(t)$ as empty sets. The detailed associating scheme is presented below.

(a) Router $v$ receiving a MLME-ASSOCIATION-REQ request from a node $u$ :

(i) If $u$ is a router and $|R(v)|<R_{\max }-\left|L_{r}(v)\right|$, then $v$ finds an index $l, 0 \leq l<R_{\max }$, such that the address $A_{u}=A_{v}+C_{\text {skip }}\left(d_{v}\right) \cdot l+1$ is free, and assigns the address $A_{u}$ to $u$ by replying a $M L M E$ ASSOCIATION response with $A_{v}, d_{v}$ and $s_{v}$ to $u$. Then $v$ updates $R(v)$ as $R(v) \cup\left\{A_{u}\right\}$.

(ii) If $u$ is an end device and $|E(v)|<C_{\max }-R_{\max }-$ $\left|L_{e}(v)\right|$, then $v$ finds an index $l, 0 \leq l<C_{\max }-$ $R_{\text {max }}$, such that the address $A_{u}=A_{v}+R_{\text {max }}$. $C_{\text {skip }}\left(d_{v}\right)+l+1$ is free, and assigns $A_{u}$ to $u$ by replying a $M L M E-A S S O C I A T I O N$ response with $A_{v}, d_{v}$ and $s_{v}$ to $u$. Then $v$ updates $E(v)$ as $E(v) \cup$ $\left\{A_{u}\right\}$.

(iii) Otherwise, if $\left|B_{r}(v)\right|+\left|B_{e}(v)\right| \leq B_{\max }$ and $s_{v}=$ "original", $v$ notifies $u$ its $d_{v}$ and asks $u$ to issue a request Borrowing-REQ if $u$ desires to connect to the network.

(iv) If steps (i), (ii) and (iii) all fail, $v$ ignores the request.

(b) Node $u$ receiving a $M L M E-A S S O C I A T I O N$ response containing $d_{v}$ and $s_{v}$ from node $v$ : Node $u$ updates its network address $A_{u}$, sets $p(u)$ as $A_{v}, d_{u}$ as $d_{v}+1$ and $s_{u}$ as $s_{v}$, and joins as $v$ 's child.

(c) Node $u$ receiving a response from $v$ containing $d_{v}$ referring $u$ to issue a response of sending a request Borrowing-REQ:

(i) If $u$ is a FFD, then it sends an Orphan-Count-REQ with value $d=\min \left\{L_{\max }-\left(d_{v}-2\right), L_{\max }-1\right\}$ to its $d$-hop neighbors to compute $o(u)$ by accumulating Orphan-REPs. Then it sets $o(u)$ as $o(u)+1$ and sends a Borrowing-REQ with the desired address space of $o(u)$ to $v$.

(ii) If $u$ is a RFD, it sends a Borrowing-REQ with the desired address space of $o(u)=0$ to $v$.

(d) Node $x$ receiving an Orphan-Count-REQ with a value $d$ : If $x$ is an orphan node and it is the first time that $x$ receives this packet, then $x$ sends a Orphan-REP to the source node first and $x$ rebroadcasts an Orphan-Count$R E Q$ to its neighbors with the value $d+1$ if $x$ is a FFD and $d+1<L_{\max }$. Otherwise, $x$ ignores the request.

(e) Router $v$ receiving a Borrowing-REQ with the desired address space of $o(u)$ from a node $u$ :

(i) Router $v$ sends an $A S K-A D D-R E Q$ with the value $h=0$ and the value $o(u)$ to its ancestors and descendants which are in $v$ 's 2-hop neighbors.

(ii) When router $v$ receiving $A S K-A D D-R E P s$ with free address spaces, $v$ selects one whose free address space $f$ is the closest to $o(u)$ and then updates $B_{r}(v)$ if $u$ is a FFD $(o(u)>1)$. If $u$ is a RFD $(o(u)=0)$, it selects the address whose depth is the smallest and updates $B_{e}(v)$.

(iii) Then $v$ sends this address or address space with its depth by replying a MLME-ASSOCIATION response with $A_{v}, d_{v}$ and the value $s_{v}=$ "borrowed" to $u$ and sends an $A S K-A D D$-Confirm with the borrowing address $A_{y}$ to the lender.

(f) Router $x$ receiving an $A S K-A D D R-R E Q$ with the value $h$ and the value $o(u)$ :

(i) If it is the first time that $x$ receives this packet and $s_{x}=$ "original", then $x$ checks its address space and rebroadcasts an $A S K-A D D R-R E Q$ to its neighbors with the value $h+1$ if $h+1<2$.

(1) If $o(u)>1$ and $|R(v)|<R_{\max }-\left|L_{r}(v)\right|$, then $x$ finds an index $l, 0 \leq l<R_{\max }$, such that the address $A_{y}=A_{x}+C_{s k i p}\left(d_{x}\right) \cdot l+1$ is free, and replies an $A S K-A D D-R E P$ with $d_{x}, A_{y}$, and the value $f=C_{\text {skip }}\left(d_{x}\right)$ to the source node.

(2) If $o(u)=0$ and $|E(x)|<C_{\max }-R_{\max }-$ $\left|L_{e}(x)\right|$, then $x$ finds an index $l, 0 \leq l<$ $C_{\max }-R_{\max }$, such that the address $A_{y}=$ $A_{x}+R_{\text {max }} \cdot C_{\text {skip }}\left(d_{x}\right)+l+1$ is free, and replies an $A S K-A D D-R E P$ with $d_{x}, A_{y}$, and the value $f=0$ to the source node. 
(ii) Otherwise, $x$ ignores the request.

(g) Router $x$ receiving an $A S K-A D D$-Confirm with the lending address $A_{y}$ from router $v$ : Router $x$ updates $L_{r}(x)$ if this address would be assigned to a child router; otherwise, it updates $L_{e}(x)$.

In the example of Fig. 1(b), $z$ lends address 36 to $y$, so its lend list $L_{r}(z)=\{(36, y=54)\}$. On the other hand, $y$ should update its borrow list $B_{r}(y)=\{(36, z=1,1)\}$. Fig. 3 shows the flow chart for an orphan node $x$ to request a router node $v$ to lend an address block to serve it.

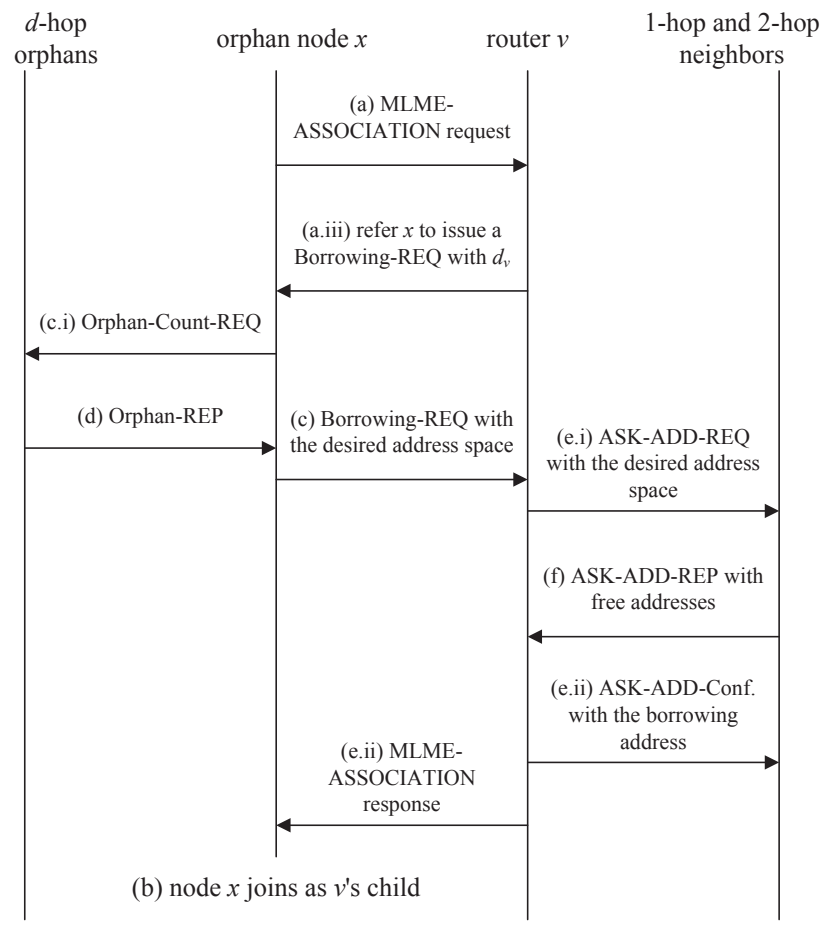

Fig. 3. The flow chart of borrowing address.

Next, we analyze the depth of the ZigBee tree after borrowing. Since we do not allow recursive borrowing and a node can only inquire its 2-hop neighbors for borrowing, the maximum depth of the ZigBee tree formed by ABS is $L_{\max }+2$.

3) Disassociation Scheme: When a node wants to disassociate with its parent router, it should broadcast a MLMEDISASSOCIATION request to its parent and descendants to ensure that its address block can be re-used and its descendants can try to reassociate with other parents. The detailed disassociating scheme is presented below.

- Node $v$ receiving a MLME-DISASSOCIATION request from node $u$ :

(a) If $u$ is $v$ 's child, then $v$ removes $u$ from $R(v), E(v)$, $B_{r}(v)$, and $B_{e}(v)$. If this address is a borrowed one, then $v$ sends a Return-ADD with the address $A_{u}$ to its original lender. This address $A_{u}$ then can be reused by other nodes.

(b) If $u$ is $v$ 's parent, then $v$ sends a MLMEDISASSOCIATION request to its children. Since $v$ is not an orphan, it should try to reassociate with the ZigBee network.

(c) Otherwise, $v$ ignores the request.

- Router $x$ receiving a Return-ADD with $A_{u}$ from node $v$ : Then $x$ removes $A_{u}$ from $L_{r}(x)$ and $L_{e}(x)$.

Fig. 4 shows the flow chart of the above procedure.

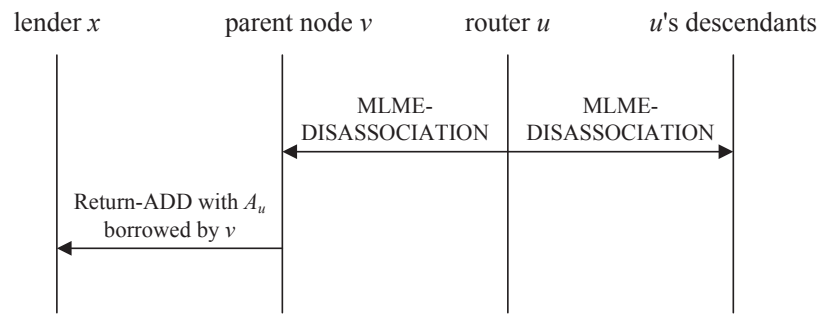

Fig. 4. The flow chart of disassociation and address returning.

\section{Routing Scheme with Address Borrowing}

With the above address borrowing scheme, ZigBee can still support very simple address-based routing as follows. When router $v$ receives a packet with a destination address $A_{\text {dest }}$, it accepts the packet if $A_{v}=A_{d e s t}$. Otherwise, $v$ forwards this packet as follows.

1) If dest is a child router or grandchild of $v$, then $v$ forwards this packet to a router as follows.

a) If $A_{v}<A_{\text {dest }} \leq A_{v}+R_{\max } \cdot C_{\text {skip }}\left(d_{v}\right)$, $v$ calculates $A_{r}$ as $A_{v}+1+\left\lfloor\frac{A_{d e s t}-\left(A_{v}+1\right)}{C_{s k i p}\left(d_{v}\right)}\right\rfloor \cdot C_{\text {skip }}\left(d_{v}\right)$. Then $v$ forwards this packet to its child router $A_{r}$ if $A_{r} \notin L_{r}(v)$; otherwise, $v$ forwards this packet to the borrower who borrows $A_{r}$ from $v$.

b) If $A_{x} \leq A_{\text {dest }} \leq A_{x}+R_{\max } \cdot C_{\text {skip }}\left(d_{x}\right)$ for some node $x$ in $B_{r}(v), v$ forwards this packet to its child router $x$.

2) If dest is a child end device, then $v$ forwards this packet to one of its child end device as follows.

a) If $A_{v}+R_{\text {max }} \cdot C_{\text {skip }}\left(d_{v}\right)<A_{\text {dest }}<A_{v}+$ $C_{\text {skip }}\left(d_{v}-1\right), v$ forwards this packet to its child end device $A_{\text {dest }}$ if $A_{\text {dest }} \notin L_{e}(v)$; otherwise, $v$ forwards this packet to the borrower who borrows $A_{r}$ from $v$.

b) If $A_{\text {dest }} \in B_{e}(v), v$ forwards the packet to this child end device.

3) Otherwise, $v$ forwards the packet to its parent $p(v)$ if $v$ is not the coordinator, and $v$ ignores this packet if $v$ is the coordinator.

Fig. 5 is an example that a packet is transmitted from node 108 to node 45 . This packet is transmitted to node 0 in accordance with the step 3) of the routing scheme first. Then node 0 forwards it to node 1 according to the step 1.a) of the routing scheme. Node 1 finds that address 45 should be one of its grandchildren, and this address is lent to node 54 . According to the step 1.a), node 1 then forwards this packet to node 54 by looking address 45 up in the lend list of node 


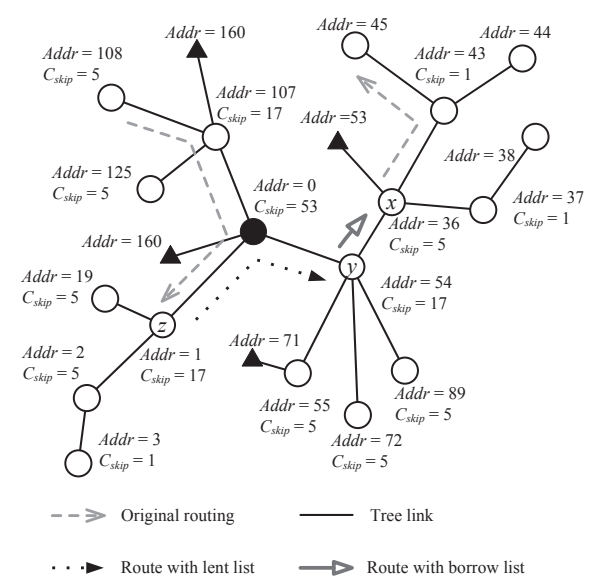

Fig. 5. An example of the routing transmitted from node 108 to node 45 on the ZigBee tree formed by ABS.

1. When node 54 receives the packet, it looks this address up in the borrow list and then forwards it to the child router 36 according to the step 1. b). Finally node 36 forwards this packet to node 45 in accordance with the step 1.a).

\section{Simulation Results}

To verify the benefit gained from our ABS, we evaluate the average number of orphans of our scheme as compared to other approaches. We assume the following simulation environment. The monitoring region is $500 \times 500 \mathrm{~m}^{2}$ where 2000 routers are randomly deployed and the coordinator is set in the center. The communication range of each router is set to $50 \mathrm{~m}$.

For comparison, we design three modifications of our ABS by relaxing the borrowing strategy in the step (e) of associating scheme. A parent router can ask for borrowing from its 1hop neighbors (ABS-1), 2-hop neighbors (ABS-2), and 3hop neighbors (ABS-3). We also set DBAS-1 and DBAS-2 as the strategies of asking its 1-hop, and 2-hop neighbors, respectively.

We vary the maximum network depth $\left(L_{\max }\right)$ to see how this influences the average number of orphans. We set $B_{\max }=2$. Fig. 6(a) shows the results. The average number of orphans is inversely proportional to the tree depth limit $L_{\max }$. Regardless of $L_{\max }$, our schemes always outperform other approaches. The average number of orphans by ABS-2 is fewer than that by ABS-1, since ABS-2 can serve further nodes.

Fig. 6(b) shows the average number of orphans with different $R_{\max }$. Regardless of borrowing strategy, the higher $R_{\max }=C_{\max }$ makes nodes join the network more easily, and thus fewer orphans.

ABS always outperform DBAS, because it allows a parent router to borrow a flexible address space through counting orphans. On the contrary, DBAS always lets a parent router borrow a maximum address space, exhausting address quickly.

Finally, we measure the impact of $B_{\max }$ on the average number of orphans. $B_{\max }$ affects the number of children that

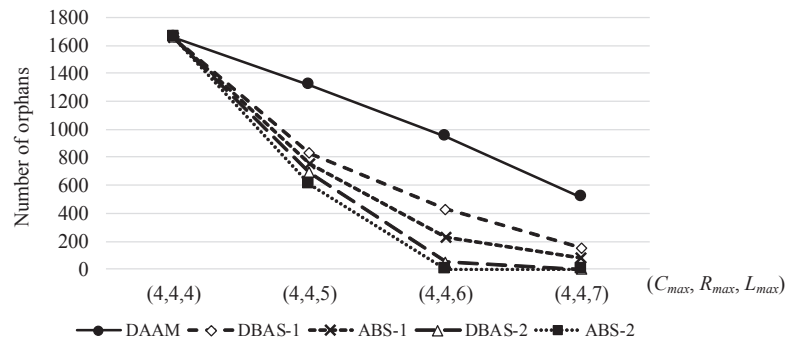

(a)

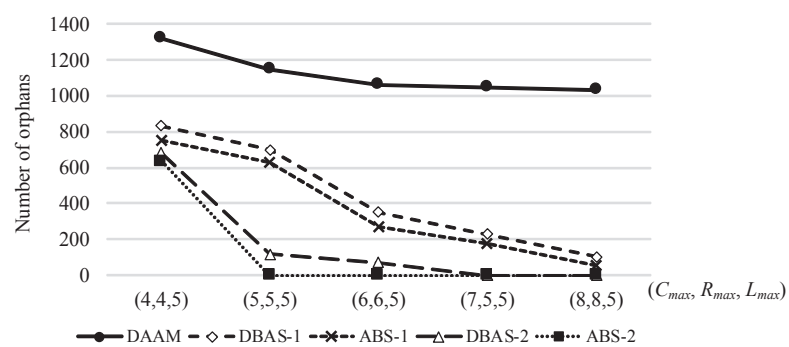

(b)

Fig. 6. The average number of orphans with different $R_{\max }$.

a router can serve in the ZigBee tree. In Fig. 7, we observe that larger $B_{\max }$ cause fewer orphans.

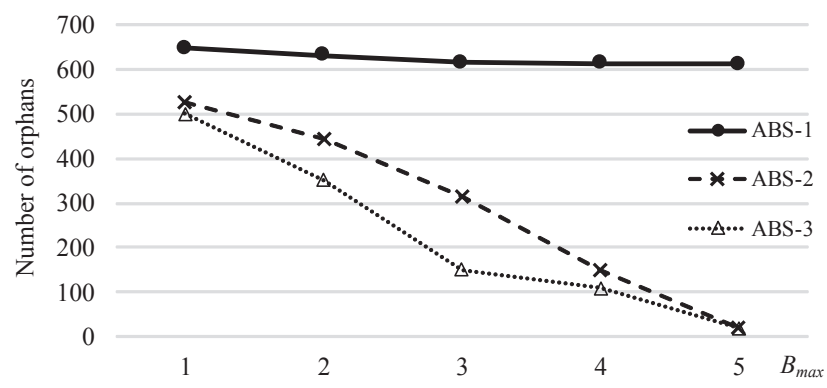

Fig. 7. The average number of orphans with different $B_{\max }$.

\section{CONCLUSions}

To relieve the orphan problem in a ZigBee network, we have proposed the address-borrowing scheme (ABS) by allowing a node to borrow unused address spaces from neighbor nodes. We have shown that it effectively decreases the number of orphans in a ZigBee network when the network cannot expand due to the constraints of $C_{\max }, R_{\max }$, and/or $L_{\max }$. The unit of lending/borrowing is a subtree of address space under the original definition in ZigBee, thus reducing the cost of routing tables and the requirement of storage spaces in router nodes. We have also suggested a light-weight routing algorithm for ABS which follows the original ZigBee address-based routing strategy with a slight modification. In addition, the maximum depth of the ZigBee network is predictable. Simulation results also show that ABS can befit original ZigBee address assignment mechanism and thus effectively reduces orphans. 


\section{ACKNOWLEDGEMENT}

Y.-C. Tseng's research is co-sponsored by MoE ATU Plan, NSC 101-2221-E-009-024-MY3, NSC 102-2218-E-009-002, IVF-NSC joint research grant 21280013 (102-2923-E-009001-MY2), Academia Sinica AS-102-TP-A06, ITRI, hTC, Delta, Proscend, and D-Link.

\section{REFERENCES}

[1] H.-S. Ahn and K. H. Ko. Simple Pedestrian Localization Algorithms Based on Distributed Wireless Sensor Networks. IEEE Trans. on Industrial Electronics, 56(10):4296-4302, 2009.

[2] A. Chen, T. H. Lai, and D. Xuan. Measuring and Guaranteeing Quality of Barrier-Coverage in Wireless Sensor Networks. In Proc. of ACM Int'l Symposium on Mobile Ad Hoc Networking and Computing (MobiHoc), 2008.

[3] M. Fang, J. Wan, and X. Xu. A Preemptive Distributed Address Assignment Mechanism Wireless Sensor Networks. In Proc. of WiCOM '08. 4th International Conference on Wireless Communications, Networking and Mobile Computing, pages 1-5, 2008.

[4] IEEE standard for information technology - telecommunications and information exchange between systems - local and metropolitan area networks specific requirements part 15.4: wireless medium access control (MAC) and physical layer (PHY) specifications for low-rate wireless personal area networks (LR-WPANs), 2003.

[5] IEEE standard for information technology - telecommunications and information exchange between systems - local and metropolitan area networks specific requirements part 15.4: wireless medium access control (MAC) and physical layer (PHY) specifications for low-rate wireless personal area networks (LR-WPANs)(revision of IEEE Std 802.15.42003), 2006

[6] F. Kuhn, R. Wattenhofer, and A. Zollinger. An Algorithmic Approach to Geographic Routing in Ad Hoc and Sensor Networks. IEEE/ACM Trans. on Networking, 16(1):51-62, 2008.

[7] M.-S. Pan, C.-H. Tsai, and Y.-C. Tseng. The Orphan Problem in ZigBee Wireless Networks. IEEE Trans. on Mobile Computing, 8(11):1573$1584,2009$.

[8] S. Park, E. J. Lee, J. H. Ryu, S.-S. Joo, and H. S. Kim. Distributed borrowing addressing scheme for ZigBee/IEEE 802.15.4 wireless sensor networks. ETRI Journal, 31(5):525-533, 2009.

[9] F. Salvadori, M. de Campos, P. S. Sausen, R. F. de Camargo, C. Gehrke, C. Rech, M. A. Spohn, and A. C. Oliveira. Monitoring in Industrial Systems Using Wireless Sensor Network with Dynamic Power Management. IEEE Trans. on Instrumentation and Measurement, 58(9):31043111, 2009.

[10] ZigBee-2011 specification, ZigBee document 053474r19, 2011. 